\title{
Facile Preparation of Silk Fibroin Scaffold Via Direct Solvent Exchange ${ }^{\star}$
}

\author{
Qiu-Sheng Wang, Guo-Cong Han, Shu-Qin Yan, \\ Ren-Chuan You, Qiang Zhang* \\ Key Laboratory of Textile Fiber \& Product (Ministry of Education), School of Textile Science and \\ Engineering, Wuhan Textile University, Wuhan 430200, China
}

\begin{abstract}
Although silk fibroin (SF) materials have gained extensive attention in tissue engineering due to their good machinability, biocompatibility, biodegradability, the complex processes, unmatched pore structures, and chemical crosslinker still hinder their mass production and clinic use. In this study, we reported a direct green and high-efficiency process to fabricate 3D silk fibroin scaffold by solvent exchange in water. The pore parameters were easily regulated with $\mathrm{NaCl}$ as auxiliary porogen. More importantly, without using any crosslinker or organic chemical-induced crystallin, SF scaffolds is mainly constructed with stable silk II crystalline (constituent with $\beta$-sheets), which was confirmed by Flourier transform infrared spectroscopy (FTIR) and X-ray diffraction (XRD). Additionally, the scaffold in our research keeps good tunability on mechanical properties, which has been demonstrated by the results of mechanical testing and provides a feasible way to optimize physical cues for further applications. Thus, the 3D porous silk scaffold with high efficiency and promising structures broaden the potential as a substitute for biomaterials.
\end{abstract}

Keywords: Silk Fibroin; Formic Acid; Solvent Exchange; Porous Materials

\section{Introduction}

In tissue engineering, The scaffolds play a significant role to provide three-dimensional templates and synthetic extracellular matrix (ECM) environment for tissue regeneration [1]. In addition to mimic the 3D geometry for tissue engineering, the porous materials should provide a microenvironment for cell attachment, differentiation, and proliferation [2-5]. Therefore, the qualified scaffolds have to meet some attributes, such as chemical composition, physical structure, and biological function.

${ }^{\star}$ Project supported by the Nature Science Foundation of Hubei Province, China (2017CFB578,184 2018CFB663), National Nature Science Foundation of China (51403163, 51303141, and 31600774).

*Corresponding author.

Email address: zhangq12041008@163.com (Qiang Zhang). 
Silk fibroin (SF) is a naturally occurring polymer, which has been widely used and investigated for centuries [6]. In particular, SF has been drawing many attention on biomaterial due to its biocompatibility, biodegradability, controllable mechanical properties, and versatility in processing into multiple materials formats $[7,8]$. For instance, SF can be easily processed into film, hydrogel, microsphere, and porous material, which has been widely explored as a substitute to repair the defects of native tissues [9].

How to increase surface area and provides a microenvironment for cell attachment and proliferation, as well as nutrient and waste exchange are the main focuses for 3D porous materials [10]. Among so many scaffolds, SF scaffolds have become an ideal substitute for tissue repairing and regeneration. In order to fabricate SF scaffold, various fabrication strategies have been developed, including salt leaching, lyophilization, freeze-thaw treatments, gas foaming, electrospinning, and 3D printing [11-16]. Nevertheless, it is the unmatched characteristics that restrict their application, including the inappropriate microstructures, fast degradation rate, and low mechanical properties. Although 3D printing provides a promising method to fabricate porous materials, the uncontrollable on micrometer level and the unmatched biological ink hinder its applications [17-19]. Additionally, the high cost, organic solvents, and complex process are remaining hinder their widely applied $[20,21]$. Thus, it's necessary to develop a new approach for preparing silk porous scaffold.

In this work, we reported a novel and effective method to fabricate 3D silk fibroin porous materials by dissolving silk into formic acid (FA) and salt filtration. As the previous reported [22, 23], silk can be dissolved in FA-Ca ${ }^{2+}$ solution system with simple operation and high dissolution efficiency. Besides, in order to satisfy the purpose that the pore structures can be tuned to match the requirements of applications, we adopt $\mathrm{NaCl}$ to adjust the pore morphology. Specifically, the morphology and microstructure of the scaffolds were characterized using scanning electron microscopy (SEM), flourier transform infrared spectroscopy (FTIR), X-ray diffraction (XRD), and the mechanical properties of SF scaffold were measured using the Texture Analyzer. Thus, this work provides a new and facile approach to produce SF scaffold efficiently.

\section{Materials and Methods}

\subsection{Preparation of SF Solution}

Regenerated BSF solution was prepared as following the procedure described previously [24]. Briefly, B. mori silk cocoons (Huzhou, Zhejiang, China) were boiled $98 \pm 2{ }^{\circ} \mathrm{C}$ three times in $0.05 \mathrm{wt} \% \mathrm{Na}_{2} \mathrm{CO}_{3}$ for 30 min to remove silk sericin, rinsed thoroughly with deionized water, and then dried at $60{ }^{\circ} \mathrm{C}$ oven overnight.

Degummed silk was dissolved in $\mathrm{FA}_{-} \mathrm{CaCl}_{2}$ (4.5wt\%) solvents, yielding a $18 \%(\mathrm{w} / \mathrm{v})$ SF-FA$\mathrm{CaCl}_{2}$ solution ${ }^{[25]}$. meanwhile, the mixture was diluted with FA to $15 \%, 12 \%, 9 \%$ (w/v). After being centrifuged at $10000 \mathrm{rpm}$ for $3 \mathrm{~min}$, the mixtures were sealed and stored at room temperature for further use.

For control groups, the degummed silk was dissolved in $9.3 \mathrm{M} \mathrm{LiBr}$ solution, by stirring at $60{ }^{\circ} \mathrm{C}$ for $2 \mathrm{~h}$. and fresh silk fibroin solution was obtained after dialysis and filtration, which was stored at $4{ }^{\circ} \mathrm{C}$ for further use. 


\subsection{Preparation of 3D Porous SF Scaffold}

The schematic diagram of the 3D porous SF scaffold preparation process is shown in Fig. 1. The different concentration $(9 \%, 12 \%, 15 \%$, and 18\%) (w/v) of SF solution were poured into cylindrically shaped container (diameter $12 \mathrm{~mm}$, height $20 \mathrm{~mm}$ ) gently until account for its $1 / 3$ volume, then adding Sodium chloride $(\mathrm{NaCl}$, particle size 200-300 $\mu \mathrm{m})$ into $\mathrm{SF}-\mathrm{FA}-\mathrm{CaCl}_{2}$ solution until fill up the whole container. Afterward, all samples were immersed in deionized water for $24 \mathrm{~h}$ to remove salt and FA completely. As for as control groups, the No. 1 control, the cylindrical shape container with $9 \%(\mathrm{~W} / \mathrm{V}) \mathrm{SF}-\mathrm{Fa}-\mathrm{Ca}^{2+}$ solution was covered and left at room temperature for 2 days, then the containers will be immersed in deionized water for 2 days to remove salt completely. the No. 2 control, $9 \%$ (W/V) SF solution was added into containers covered and left at room temperature for 5 to 7 days. All samples would be freezing-dried for further characterization.

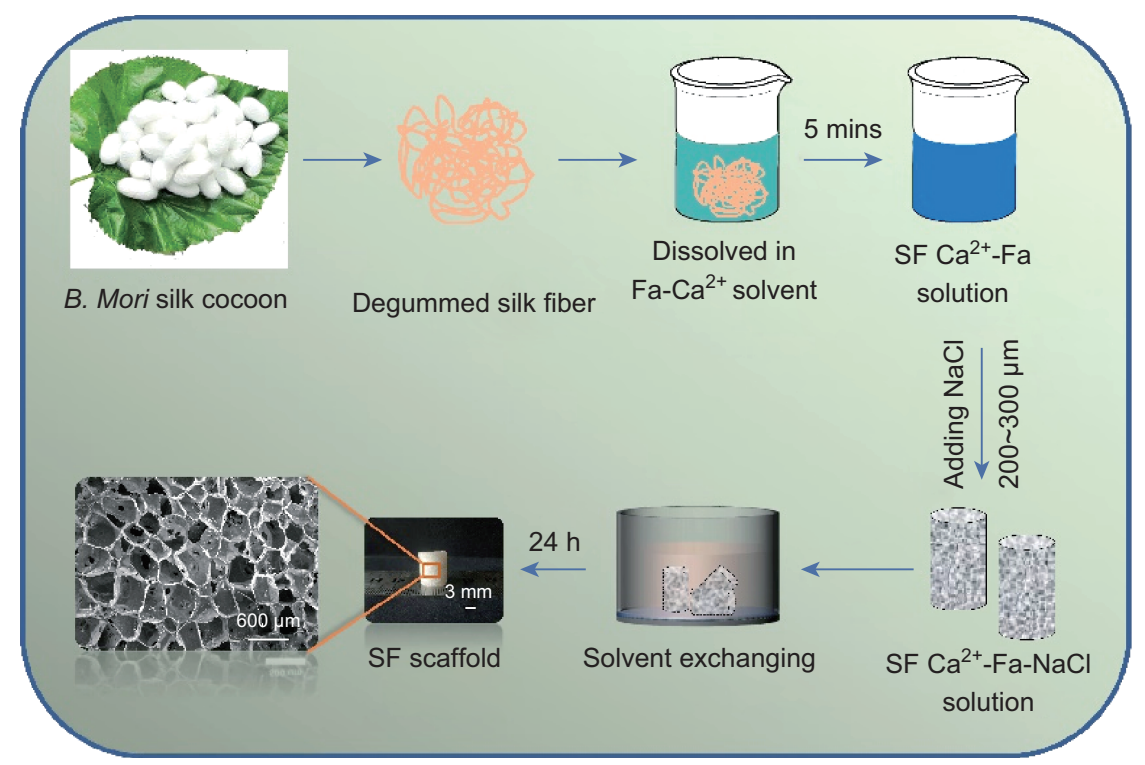

Fig. 1: Fabrication process of 3D porous SF scaffold by solvent exchange

\section{$2.3 \quad$ Structure of SF Scaffolds}

All samples were cut into micro-particles with radii less than $40 \mu \mathrm{m}$, and then prepared in $\mathrm{KBr}$ pellets. FT-IR spectra were obtained in the range of $400-4000 \mathrm{~cm}^{-1}$ with an accumulation of 64 scans and a resolution of $4 \mathrm{~cm}^{-1}$ with a VERTEX 70 [26].

$\mathrm{X}$-ray diffraction of the sample of SF porous materials was performed with Ni-filtered $\mathrm{Cu}-\mathrm{Ka}$ radiation $\left(\lambda=0.15418 \mathrm{~nm}\right.$ ) from the X-ray generator ( $X^{\prime}$ Pert PRO) at $40 \mathrm{kV}$ and $40 \mathrm{~mA}$ X-ray diffraction. And the scanning speed was $2^{\circ} / \mathrm{min}$. Diffraction intensity curves with $2 \theta$ from $5^{\circ}$ to $45^{\circ}$ were obtained [27].

\subsection{Pore Morphology}

The cross-sectional morphology of all samples were observed using a scanning electron microscope (SEM; JEOLIT-300, Japan) as described previously [28]. The scaffolds were mounted on a copper 
plate and sputter-coated with a gold layer of 20-30 nm thickness prior to imaging. The border of each pore in the top layer was defined according to a gradient method. The images of the top layer of the cross-sections were obtained. Additionally, the diameter distribution of different scaffold was analyzed by the software of Nano Measures 1.2, over 100 pores diameters were collected for each different scaffold.

\subsection{Mechanical Properties}

The mechanical properties of the SF scaffolds in wet condition were measured according to the investigation [29]. Before measurement, the samples were cut into suitable size (12 $\mathrm{mm}$ in diameter and $10 \mathrm{~mm}$ in height) and then immersed in distilled water for $4 \mathrm{hs}$. Then the Texture Analyzer (TMS-PRO, Food technology corporation) was used to compress the samples with a 25 $\mathrm{N}$ loading cell at room temperature. The cross-head speed kept $5 \mathrm{~mm} \mathrm{~min}^{-1}$ until the compression deformation of the samples was over $80 \%$ of its original length. The linear-elastic region was cut from the stress-strain curves to calculate the compressive modulus of the samples, and four samples were carried out the same test for each group.

\section{Statistical Analysis}

Data are presented as means \pm SD. Statistical comparisons were performed using ANOVA, and differences at $P<0.05(*)$ were considered statistically significant.

\section{$3 \quad$ Results and Discussions}

\subsection{Second Structure of 3D Porous SF Scaffolds}

Previous studies about SF have revealed that the characteristic absorption bands at $1650-1655$ $\mathrm{cm}^{-1}$ (amide I), $1525-1540 \mathrm{~cm}^{-1}$ (amide II), $1266 \mathrm{~cm}^{-1}$ (amide III), and $669 \mathrm{~cm}^{-1}$ (amide V) were assigned to $\alpha$-form, and $\beta$-sheets have characteristic peaks at $1620-1635 \mathrm{~cm}^{-1}$ (amide I), $1530 \mathrm{~cm}^{-1}$ (amide II), $1230-1235 \mathrm{~cm}^{-1}$ (amide III), and $700 \mathrm{~cm}^{-1}$ (amide V), and absorbance peaks at $1655-1660 \mathrm{~cm}^{-1}$ (amide I), $1535-1545 \mathrm{~cm}^{-1}$ (amide II), $1235 \mathrm{~cm}^{-1}$ (amide III), and $650 \mathrm{~cm}^{-1}$ (amide V) were assigned to the random coil conformation ${ }^{[30,31]}$. On another hand, the crystal structure was studied with $\mathrm{Cu} K_{\alpha}$ radiation, which revealed that the main diffraction peaks of the silk I crystal occur at $12.2^{\circ}(d=7.25 \AA$, medium strong $), 19.7^{\circ}(d=4.5 \AA$, strong), $24.7^{\circ}(d=3.60 \AA$, medium $)$, and $28.2^{\circ}(d=3.16 \AA$, medium $)$, and the silk II occur at $9.1^{\circ}$ $(d=9.7 \AA$, medium strong $), 18.9^{\circ}(d=4.69 \AA$, medium strong $)$ and $20.7^{\circ}(d=4.30 \AA$, very strong) $[30,32]$.

In this study, FTIR and XRD measurements of SF scaffold with different concentration and processing methods were performed to determine their second structures. As shown in Fig. 2(A), the SF scaffold prepared from SF-FA-CaCl $\mathrm{Cl}_{2}$ and $\mathrm{SF}$ in aqueous solution showed absorption bands of $\beta$-sheets at $1701 \mathrm{~cm}^{-1}$ and $1625 \mathrm{~cm}^{-1}$, which have the similarly or same bands with the control groups. For XRD curves (Fig. 2(B)), a major peak at $20.7^{\circ}$, a weak peak at $24.3^{\circ}$, and a broad peak at $9.1^{\circ}$ were observed, which representing silk II crystalline structure for all SF porous materials. Obviously, the scaffold via solvent exchange show an almost second structures with the 

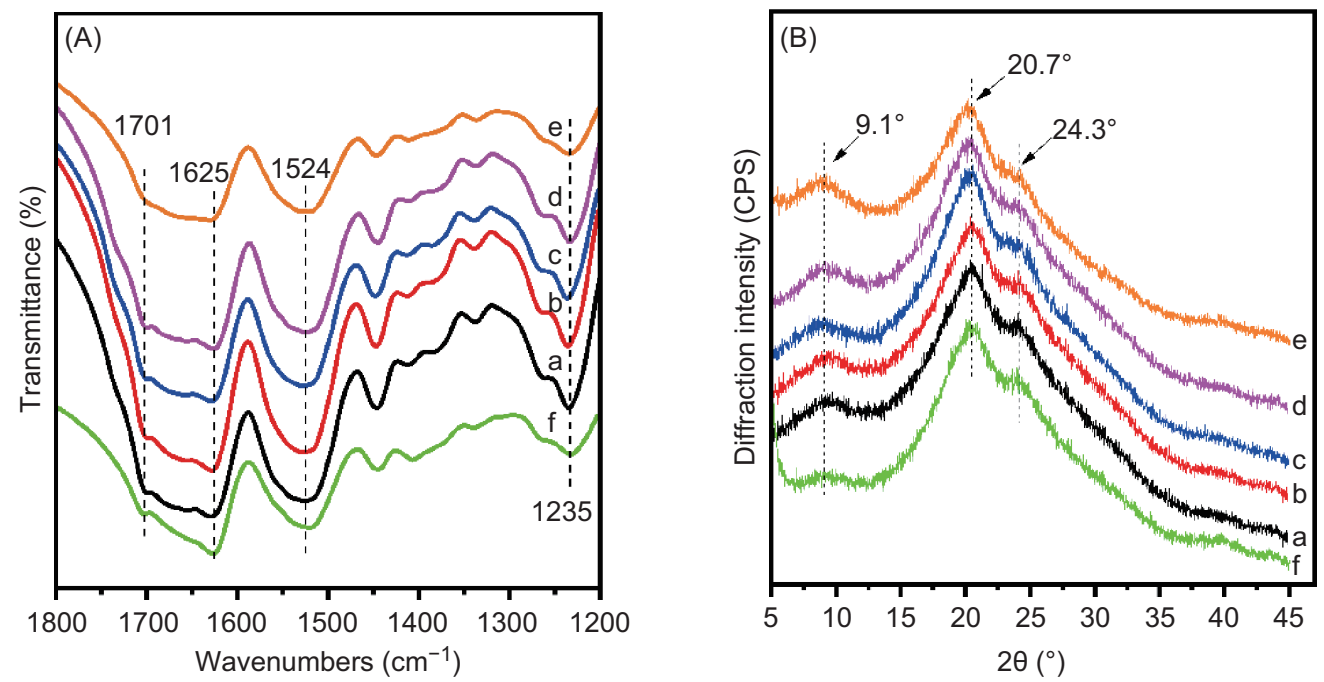

Fig. 2: FTIR spectroscopy and X-ray Diffraction Curve of 3D porous SF scaffold (a 9\%, b 12\%, c 15\%, d $18 \%$ (w/v) SF-FA-Ca ${ }^{2+}$; e the control No. 1 group; f the control No. 2 group)

conventional method to prepare SF scaffold. Especially, these $\beta$-sheets and silk II components would contribute to stable morphology and enough mechanical properties of the SF 3D porous materials for biological applications.

\subsection{Morphology of SF Scaffolds}

The SEM results in Fig. 3 revealed that there are significant differences between the experimental group and the control group. Obviously, the SF scaffold (Fig. 3(A)-3(D)) of experimental groups show well defined 3D structure with a distinctive boundary, and the pores are interconnected by a number of small pores in the walls, which will benefit to nutrient and waste exchange [33, 34]. Additionally, as shown in Fig. 3(H), a more uniform pore size (around $300 \mu \mathrm{m}$ ) was observed with little influence on the various concentration of SF, which was likely due to the particle size of $\mathrm{NaCl}$ sieved $(200 \sim 300 \mu \mathrm{m})$. As control groups (Fig. 3(e), 3(f)), the irregular pore structure is a barrier for the pore morphology tuned and nutrition-waste exchanged. Higher magnification images (Fig. 3(a)-3(f)) showed that the 3D porous SF scaffold retained a relatively smooth surface. Therefore, it is an effective method to fabricate SF 3D porous materials by the new dissolving system to meet the acquirements for cells attachment and proliferation.

\subsection{Compressive Mechanical Properties of SF Scaffolds}

One of the major requirements of scaffold is the mechanical properties [35]. Fig. 4(A) is the stress-strain curve of SF scaffold. Like the other polymers, the stress-strain curve was manifested as a remarkable nonlinear relationship, which showed that the ability of the SF scaffold to resist the deformation gradually increase. As shown in stress-strain curve, the action of the external deformation process can be divided into three stages: linear starting section $(0-40 \%)$, steep rise section (40-80\%), and irreversible stage. Specificity, At the first stage, a wide deformation required external force was very small due to the deformation of pore structure. At the second stage, the external force increased sharply, which corresponding to the elastic deformation of 

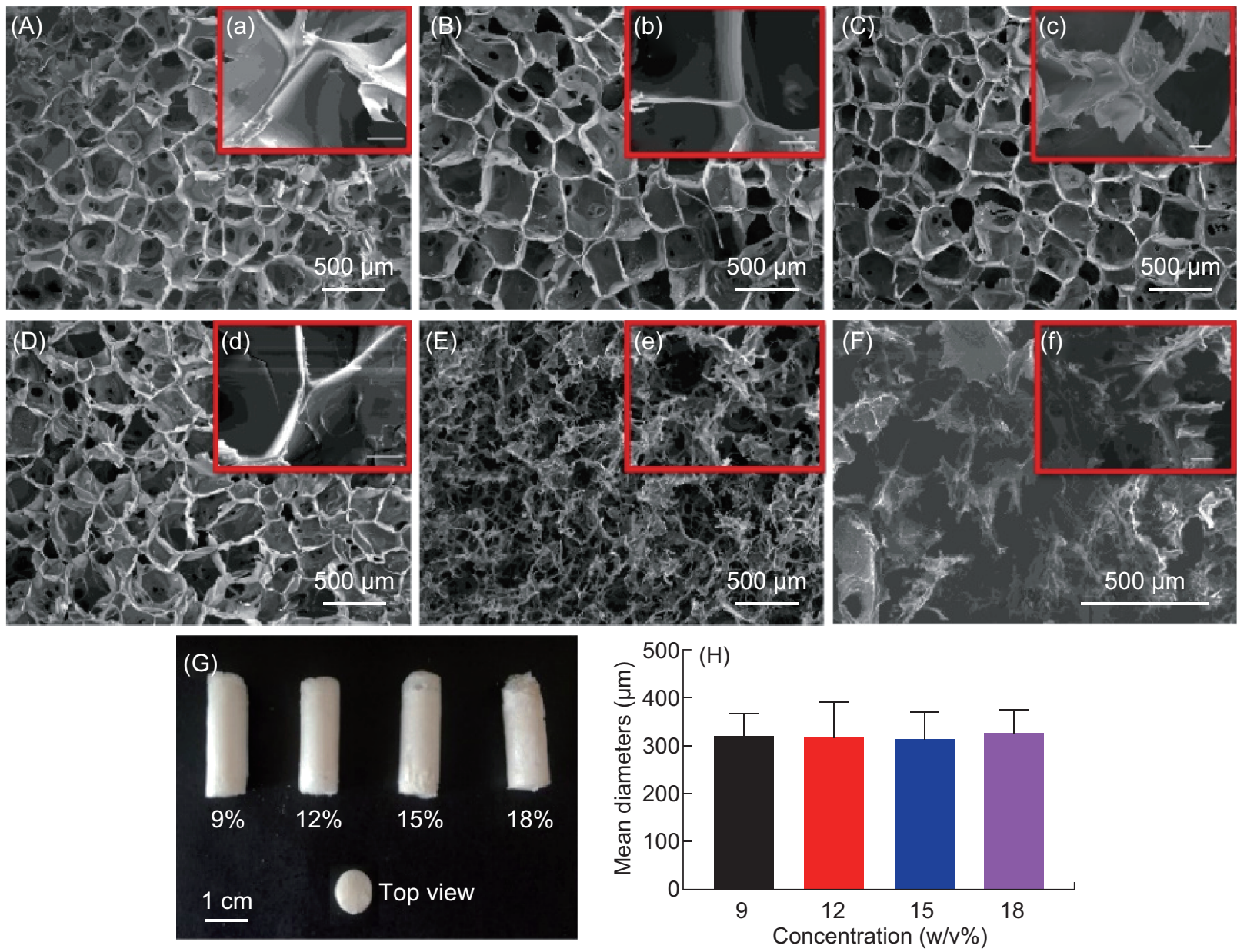

Fig. 3: SEM images, photo, and the pore diameters of 3D porous SF scaffold cross section ((A) 9\%, (B) $12 \%,(\mathrm{C}) 15 \%,(\mathrm{D}) 18 \%$ (w/v) SF-FA-Ca ${ }^{2+}$; (E) the control No. 1 group; (F) the control No. 2 group, (a)-(f) magnified images of image (A)-(F); scale bar: (A)-(F): $500 \mu \mathrm{m}$; (a)-(f): $50 \mu \mathrm{m}$; (G) $1 \mathrm{~cm}$ )

the pore wall. At the last stage, irreversible damage happened with the deformation increased furtherly, it means the scaffold couldn't revert to its original shape. Compared with the control groups, the 3D SF scaffold could maintain a larger deformation with a suitable loading, which provides a huge potential to be applied in cartilage tissue.

Fig. 4(B) showed the compressive modulus of different SF scaffolds. There is a remarkable difference between the control No. 1 group and $9 \%(\mathrm{~W} / \mathrm{V})$ porous SF scaffold, to be exact, the compressive modulus of the former $(309.35 \pm 67.56 \mathrm{KPa})$ was three times higher than the later $(94.45 \pm 34.24 \mathrm{KPa})$. This mainly because the larger pore size is more sensitive for small external pressure than small pore size structure. However, based on the same pore size, the compressive modulus increased slightly with the concentration of SF raised, which means the mechanical properties of 3D porous SF scaffold can be tuned to some extent.

It is encouraged for 3D porous SF scaffold prepared with a facile operation, high efficiency, and good reproducibility. In this study, silk fibers dissolved in $\mathrm{FA}_{-} \mathrm{Ca}^{2+}$ solution was much simpler and more rapidly than $\mathrm{LiBr}$ system process. Furthermore, the pore size of SF scaffold can be tuned by the diameters of $\mathrm{NaCl}$ as porogen. More importantly, a stable silk-II structure can be detected by $\mathrm{XRD}$, and without adding any organic solvent in this process, these mainly because 

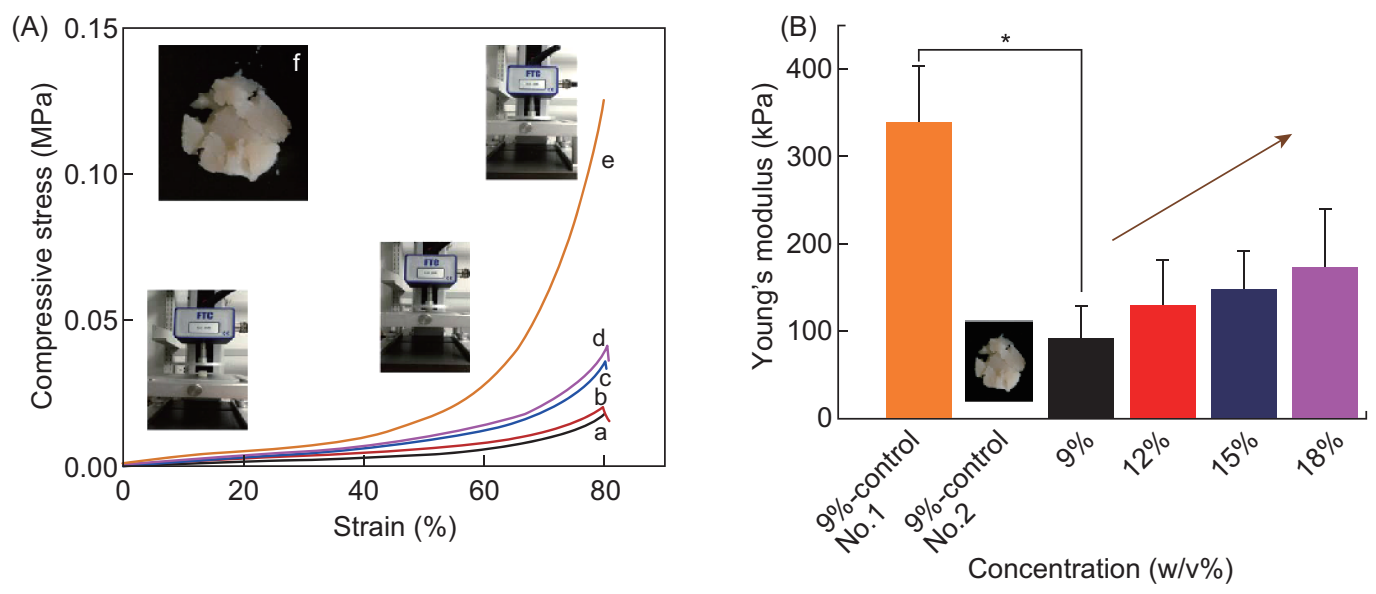

Fig. 4: Compressive stress-strain curve and compressive modulus of 3D porous SF scaffold in wet state (a $9 \%$, b 12\%, c 15\%, d 18\% (w/v) SF-FA-Ca ${ }^{2+}$; e, the control No. 1 group; f, the control No. 2 group). Data are presented as mean \pm standard error $(n=5, P<0.05)$

silk was swollen to fibril structure in $\mathrm{Ca}^{2+}$ and formed the random coil structure in FA solution, once immersed in water, it is easier to aggregate into crystalline $\beta$-sheet for fibrils after $\mathrm{Ca}^{2+}$ removed and the $\mathrm{pH}$ value increased to isoelectric point of silk proteins $(\mathrm{pI} \approx 4.2)$ during the solvent exchange process (Fig. 5). In addition, the regulatability of mechanical properties and pore structure provided a possibility for satisfying the requirements of different tissues.

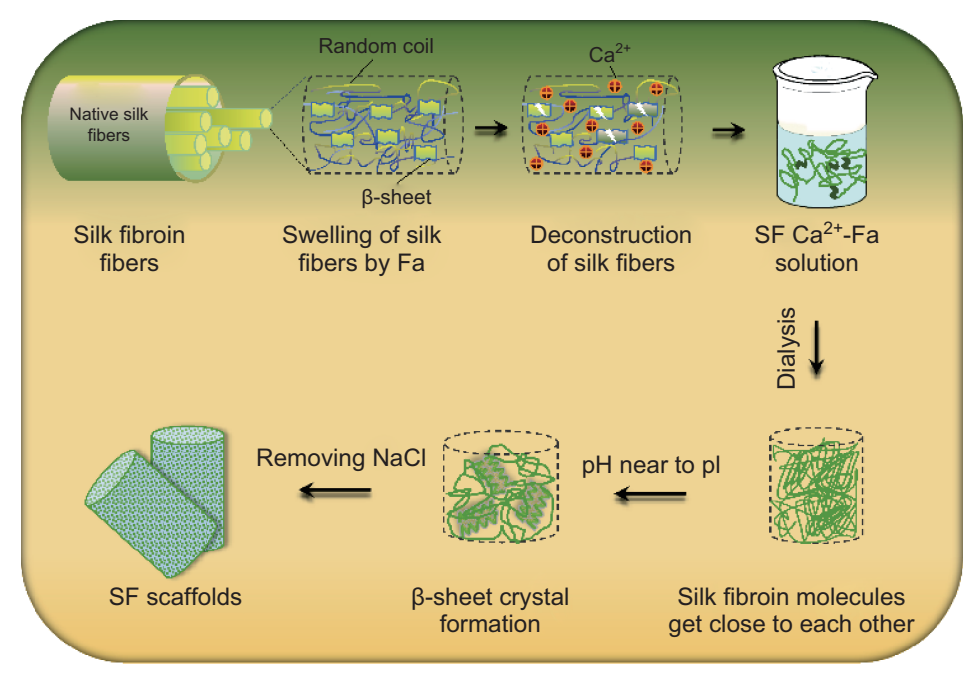

Fig. 5: The mechanism of silk scaffolds formation based on solvent exchange

\section{Conclusions}

The 3D porous SF scaffolds were successfully prepared via solvent exchange directly. More importantly, this efficient process for fabrication of SF scaffold without using of high-cost crosslinker and hazardous physical treatment compared to conventional SF scaffold. Additionally, the $\beta$ sheet and silk II crystalline structures ensure the stable morphology and adequate mechanical properties of $\mathrm{SF}$ scaffold. Adjusting the diameters of $\mathrm{NaCl}$ is a feasible way to regulate the pore 
morphology to satisfy different tissues. Therefore, the 3D SF scaffold fabricated via solvent exchange directly offered a new and effective processing method for substitutes of biomaterials in tissue engineering.

\section{Acknowledgments}

This work was supported by the Nature Science Foundation of Hubei Province, China (2017CFB578, 2018CFB663), National Nature Science Foundation of China (51403163, 51303141, and 31600774).

\section{References}

[1] Ma P X. Biomimetic materials for tissue engineering [J]. Adv Drug Deliv Rev, 2008, 60(2): 184-98.

[2] Mandal B B, Kundu S C. Cell proliferation and migration in silk fibroin 3D scaffolds [J]. Biomaterials, 2009, 30(15): 2956-65.

[3] Mandal B B, Grinberg A, Gil E S, et al. High-strength silk protein scaffolds for bone repair [J]. Proc Natl Acad Sci U S A, 2012, 109 (20): 7699-704.

[4] Mandal B B, Park S H, Gil E S, et al. Multilayered silk scaffolds for meniscus tissue engineering [J]. Biomaterials, 2011, 32(2): 639-51.

[5] Chen G U T, Tateishi T. Scaffold Design for Tissue Engineering [J]. Macromol. Biosci., 2002, 2: 67-77.

[6] Vepari C, Kaplan D L. Silk as a Biomaterial [J]. Prog. Polym. Sci., 2007, 32(8-9): 991-1007.

[7] Melke J, Midha S, Ghosh S, et al. Silk fibroin as biomaterial for bone tissue engineering [J]. Acta Biomater., 2016, 31: 1-16.

[8] Ling S, Wang Q, Zhang D, et al. Integration of Stiff Graphene and Tough Silk for the Design and Fabrication of Versatile Electronic Materials [J]. Advanced Functional Materials, 2018, 28(9): 1705291.

[9] Han H, Ning H, Liu S, et al. Silk Biomaterials with Vascularization Capacity [J]. Adv Funct Mater, 2016, 26(3): 421-436.

[10] Xin K, Wu P, Li R, et al. Facile preparation of a novel mulberry silk fibroin scaffold for threedimensional tumor cell culture [J]. Mater. Lett., 2015, 143: 8-11.

[11] Kim U J, Park J, Kim H J, et al. Three-dimensional aqueous-derived biomaterial scaffolds from silk fibroin [J]. Biomaterials, 2005, 26(15): 2775-85.

[12] Guziewicz N, Best A, Perez-Ramirez B, et al. Lyophilized silk fibroin hydrogels for the sustained local delivery of therapeutic monoclonal antibodies [J]. Biomaterials, 2011, 32(10): 2642-50.

[13] Li X, Yan S, Qu J, et al. Soft freezing-induced self-assembly of silk fibroin for tunable gelation [J]. Int. J. Biol. Macromol., 2018, 117: 691-695.

[14] Maniglio D, Bonani W, Migliaresi C, et al. Silk fibroin porous scaffolds by N2O foaming [J]. J. Biomater. Sci. Polym. Ed., 2018, 29(5): 491-506.

[15] Ki C S, Kim J W, Hyun J H, et al. Electrospun three-dimensional silk fibroin nanofibrous scaffold [J]. JJ. Appl. Polym. Sci., 2007, 106(6): 3922-3928.

[16] Shi W, Sun M, Hu X, et al. Structurally and Functionally Optimized Silk-Fibroin-Gelatin Scaffold Using 3D Printing to Repair Cartilage Injury In Vitro and In Vivo [J]. Adv. Mater., 2017, 29(29): 1701089. 
[17] Applegate M B, Partlow B P, Coburn J, et al. Photocrosslinking of Silk Fibroin Using Riboflavin for Ocular Prostheses [J]. Adv Mater, 2016, 28(12): 2417-20.

[18] Mandrycky C, Wang Z, Kim K, et al. 3D bioprinting for engineering complex tissues [J]. Biotechnol Adv, 2016, 34(4): 422-434.

[19] Wang Q, Han G, Yan S, et al. 3D Printing of Silk Fibroin for Biomedical Applications [J]. Materials(Basel), 2019, 12(3).

[20] Li D W, Lei X, He F L, et al. Silk fibroin/chitosan scaffold with tunable properties and low inflammatory response assists the differentiation of bone marrow mesenchymal stem cells $[\mathrm{J}]$. Int J Biol Macromol, 2017, 105(Pt 1): 584-597.

[21] Lu Q, Zhang X, Hu X, et al. Green process to prepare silk fibroin/gelatin biomaterial scaffolds [J]. Macromol Biosci, 2010, 10(3): 289-98.

[22] Bie S, Ming J, Zhou Y, et al. Rapid formation of flexible silk fibroin gel-like films [J]. J. Appl. Polym. Sci., 2015, 132(15): n/a-n/a.

[23] Hwang T I, Maharjan B, Tiwari A P, et al. Facile fabrication of spongy nanofibrous scaffold for tissue engineering applications [J]. Mater. Lett., 2018, 219: 119-122.

[24] Wang Q, Yan S, Han G, et al. Facile fabrication of silk fibroin microspheres via electrostatic assembly [J]. Mater. Res. Express, 2018, 5(7): 075401.

[25] Zhang F, You X, Dou H, et al. Facile fabrication of robust silk nanofibril films via direct dissolution of silk in CaCl2-formic acid solution [J]. ACS Appl. Mater. Interfaces, 2015, 7(5): 3352-3361.

[26] Zhang Q, Chen S, You R, et al. Silk fibroin/hyaluronic acid porous scaffold for dermal wound healing [J]. Fibers and Polymers, 2017, 18(6): 1056-1063.

[27] Yan S, Wang Q, Tariq Z, et al. Facile preparation of bioactive silk fibroin/hyaluronic acid hydrogels [J]. Int. J. Biol. Macromol., 2018, 118(Pt A): 775-782.

[28] Li X, You R, Luo Z, et al. Silk fibroin scaffolds with a micro-/nano-fibrous architecture for dermal regeneration [J]. J. Mater. Chem. B, 2016, 4(17): 2903-2912.

[29] Applegate M B, Partlow B P, Coburn J, et al. Photocrosslinking of Silk Fibroin Using Riboflavin for Ocular Prostheses [J]. Adv. Mater., 2016, 28(12): 2417-2420.

[30] Zhang Q, Zhao Y, Yan S, et al. Preparation of uniaxial multichannel silk fibroin scaffolds for guiding primary neurons [J]. Acta Biomater., 2012, 8(7): 2628-2638.

[31] Lu Q, Zhang B, Li M, et al. Degradation mechanism and control of silk fibroin [J]. Biomacromolecules, 2011, 12(4): 1080-6.

[32] Wang H Y, Zhang Y Q. Processing and characterisation of a novel electropolymerized silk fibroin hydrogel membrane [J]. Sci Rep, 2014, 4: 6182.

[33] Yan S, Zhang Q, Wang J, et al. Silk fibroin/chondroitin sulfate/hyaluronic acid ternary scaffolds for dermal tissue reconstruction [J]. Acta Biomater, 2013, 9(6): 6771-82.

[34] Liu Y, Li X, Qu X, et al. The fabrication and cell culture of three-dimensional rolled scaffolds with complex micro-architectures [J]. Biofabrication, 2012, 4(1): 015004.

[35] Gil E S, Kluge J A, Rockwood D N, et al. Mechanical improvements to reinforced porous silk scaffolds [J]. J Biomed Mater Res A, 2011, 99(1): 16-28. 